\title{
Mass Renormalization in the Su-Schrieffer-Heeger Model
}

\author{
Marco Zoli \\ Istituto Nazionale Fisica della Materia - Universitá di Camerino, \\ 62032 Camerino, Italy.e-mail: zoli@campus.unicam.it
}

(October 28, 2018)

\begin{abstract}
This study of the one dimensional Su-Schrieffer-Heeger model in a weak coupling perturbative regime points out the effective mass behavior as a function of the adiabatic parameter $\omega_{\pi} / J, \omega_{\pi}$ is the zone boundary phonon energy and $J$ is the electron band hopping integral. Computation of low order diagrams shows that two phonons scattering processes become appreciable in the intermediate regime in which zone boundary phonons energetically compete with band electrons. Consistently, in the intermediate (and also moderately antiadiabatic) range the relevant mass renormalization signals the onset of a polaronic crossover whereas the electrons are essentially undressed in the fully adiabatic and antiadiabatic systems. The effective mass is roughly twice as much the bare band value in the intermediate regime while an abrupt increase (mainly related to the peculiar 1D dispersion relations) is obtained at $\omega_{\pi} \sim \sqrt{2} J$.
\end{abstract}

PACS: 63.10.+a, 63.20.Dj, 71.38.+i

A sizeable electron-phonon interaction can induce a local deformation in the lattice accompanied by the formation of a quasiparticle with multiphononic character, the polaron [1]. As the spatial extension of the lattice deformation can vary, the concepts of large and small polaron have been introduced [2 [7]: the transition between a large and a small polaron state is driven by the strength of the electron-phonon coupling [8 11] and monitored through the behavior of ground state properties such as the polaron energy band and the effective mass 112 14. In particular, an abrupt increase of the polaron mass versus the e-ph coupling is associated with the occurence of the self-trapping event and the loss of the polaron mobility properties. The aforementioned investigations of polarons generally depart from the Hamiltonian of the Holstein model [15] in which the electron couples to dispersive optical phonons [16] through a local (momentum independent) interaction while the coupling to acoustical phonons, although possible in principle [17], would lead to huge mass renormalizations [18. In fact, also the Holstein optical polaron masses are very heavy (at least larger than $10^{3}$ times the bare band mass) in the self-trapped state but masses of order 10 times the bare band mass are possible in the presence of high energy phonon spectra [19].

As an alternative to the Holstein molecular crystal model with local interactions one may consider the $\mathrm{Su}$ Schrieffer-Heeger (SSH) model Hamiltonian [20]. The tight binding SSH model was introduced to study polyacetylene [21, an essentially one dimensional polymer with delocalized $\pi$ electrons which are responsible for the alternation of double and single bonds between two neighboring carbon atoms, a dimerized state 22]. As a main characteristic of the SSH model Hamiltonian one notes that the electron-phonon interaction modifies the electron hopping matrix elements thus leading to a nonlocal (in momentum space) coupling with vertex function dependent on both the electron and the phonon wave vector. With the present paper we start an investigation 
of the ground state and finite temperature properties of systems which can be described by the SSH model. Although some references to conducting polymers are made in the introductive notation we focus here on the general features of this model which may well apply to a class of quasi one dimensional systems with short range $e-p h$ interactions [23 26]. As a preliminar goal we evaluate the relevance of the mass renormalization (induced by this non local type of electron-phonon coupling) which might provide signatures of polaron formation in the system. In 1D, the real space SSH Hamiltonian reads

$$
\begin{aligned}
H= & \sum_{r} J_{r, r+1}\left(f_{r}^{\dagger} f_{r+1}+f_{r+1}^{\dagger} f_{r}\right)+ \\
& \sum_{r}\left(\frac{p_{r}^{2}}{2 M}+\frac{K}{2}\left(u_{r}-u_{r+1}\right)^{2}\right) \\
J_{r, r+1}= & -\frac{1}{2}\left[J+\alpha\left(u_{r}-u_{r+1}\right)\right]
\end{aligned}
$$

where $J$ is the nearest neighbors hopping integral for an undistorted chain, $\alpha$ is the $\pi$ electron-phonon coupling, $u_{r}$ is the dimerization coordinate which specifies the displacement of the $(\mathrm{CH})$ group on the $r$ - lattice site along the molecular axis, $p_{r}$ is the momentum operator conjugate to $u_{r}, M$ is the $(\mathrm{CH})$ group mass, $K$ is the effective spring constant, $f_{r}^{\dagger}$ and $f_{r}$ create and destroy $\pi$ electrons on the $r-(\mathrm{CH})$ group. Incidentally we note that, by means of a Jordan-Wigner transformation, the Hamiltonian in eq.(1) maps onto that of a spin-Peierls chain [27,28]. After expanding the lattice displacement and its conjugate momentum in terms of the phonon creation and annihilation operators $b_{q}^{\dagger}$ and $b_{q}$ and Fourier transforming the electron operators, one gets the SSH Hamiltonian in momentum space:

$$
\begin{aligned}
& H=H_{0}+H_{\text {int }} \\
& H_{0}=\sum_{k} \epsilon_{k} f_{k}^{\dagger} f_{k}+\sum_{q} \omega_{q} b_{q}^{\dagger} b_{q} \\
& H_{\text {int }}=\sum_{k, q} g(k+q, k)\left(b_{-q}^{\dagger}+b_{q}\right) f_{k+q}^{\dagger} f_{k} \\
& \epsilon_{k}=-J \cos (k) \\
& \omega_{q}^{2}=4 \frac{K}{M} \sin ^{2}\left(\frac{q}{2}\right) \\
& g(k+q, k)=\frac{i \alpha}{\sqrt{2 M N \omega_{q}}}(\sin (k+q)-\sin (k))
\end{aligned}
$$

where $N$ is the total number of lattice sites. The phonon dispersion relation is defined in the range $q \in$ $[0, \pi]$. Assuming a reduced Brillouin zone $(|q| \leq \pi / 2)$ the spectrum displays both an acoustic and an optical branch. Here we attack the Hamiltonian in eq.(2) by using the Matsubara Green's functions formalism and taking the $e-p h$ term as the perturbation. Although a weak coupling perturbative approach cannot capture the full multiphononic nature of the polaronic quasiparticle, an envisaged, sizeable enhancement of the charge carrier mass even to the lowest order of perturbative theory 
would be an indicator of polaron formation. The full electron propagator is defined as:

$$
\begin{aligned}
G(p, \tau)= & -\sum_{n=0}^{\infty}(-1)^{n} \int_{0}^{\beta} d \tau_{1} \ldots d \tau_{n}\left\langle T_{\tau} f_{p}(\tau) H_{\text {int }}\left(\tau_{1}\right) .\right. \\
& \left.\cdot H_{\text {int }}\left(\tau_{n}\right) f_{p}^{\dagger}(0)\right\rangle_{0}
\end{aligned}
$$

where $\beta$ is the inverse temperature, $T_{\tau}$ is the time ordering operator, $\langle\ldots\rangle_{0}$ indicates that thermodynamic averages are taken with respect to the unperturbed Hamiltonian and only different connected diagrams contribute to any order $n$.

I have calculated the self-energy terms due to one phonon ( $n=2$ in eq.(3)) and two phonons $(n=4$ in eq.(3)) scattering processes. Their finite temperature expressions are:

$$
\begin{aligned}
\Sigma_{p}^{(1)}\left(i \varepsilon_{m}\right)= & -\sum_{q} g^{2}(p, p-q)\left[\frac{n_{B}\left(\omega_{q}\right)+n_{F}\left(-\epsilon_{p-q}\right)}{i \varepsilon_{m}-\epsilon_{p-q}-\omega_{q}}+\right. \\
& \left.\frac{n_{B}\left(\omega_{q}\right)+n_{F}\left(\epsilon_{p-q}\right)}{i \varepsilon_{m}-\epsilon_{p-q}+\omega_{q}}\right] \\
\Sigma_{p}^{(2 a)}\left(i \varepsilon_{m}\right)= & \frac{1}{\beta^{2}} \sum_{q, q_{1}} g(p, p-q) g\left(p, p-q_{1}\right) \cdot \\
& g\left(p-q, p-q-q_{1}\right) g\left(p-q_{1}, p-q-q_{1}\right) . \\
& \sum_{\omega_{n}, \omega_{l}} D_{q}^{0}\left(i \omega_{n}\right) D_{q_{1}}^{0}\left(i \omega_{l}\right) G_{p-q}^{0}\left(i \varepsilon_{m}-i \omega_{n}\right) . \\
& G_{p-q_{1}}^{0}\left(i \varepsilon_{m}-i \omega_{l}\right) G_{p-q-q_{1}}^{0}\left(i \varepsilon_{m}-i \omega_{n}-i \omega_{l}\right) \\
\Sigma_{p}^{(2 b)}\left(i \varepsilon_{m}\right)= & \frac{1}{\beta^{2}} \sum_{q, q_{1}} g^{2}(p, p-q) g^{2}\left(p-q, p-q-q_{1}\right) \cdot \\
& \sum_{\omega_{n}, \omega_{l}} D_{q}^{0}\left(i \omega_{n}\right) D_{q_{1}}^{0}\left(i \omega_{l}\right)\left[G_{p-q}^{0}\left(i \varepsilon_{m}-i \omega_{n}\right)\right]^{2} . \\
& G_{p-q-q_{1}}^{0}\left(i \varepsilon_{m}-i \omega_{n}-i \omega_{l}\right) \\
\Sigma_{p}^{(2 c)}\left(i \varepsilon_{m}\right)= & \frac{1}{\beta^{2}} \sum_{q, k} g^{2}(p, p-q) g^{2}(k, k+q) \cdot \\
& \sum_{\omega_{n}}\left[D_{q}^{0}\left(i \omega_{n}\right)\right]^{2} G_{p-q}^{0}\left(i \varepsilon_{m}-i \omega_{n}\right) \cdot \\
& \sum_{\varepsilon_{m}^{\prime}} G_{q+k}^{0}\left(i \varepsilon_{m}^{\prime}+i \omega_{n}\right) G_{k}^{0}\left(i \varepsilon_{m}^{\prime}\right) \\
&
\end{aligned}
$$

There are three contributions due to different connected two-phonons diagrams [29]. $n_{B}\left(\omega_{q}\right)$ is the Bose occupation factor and $n_{F}\left(\epsilon_{p}\right)$ is the Fermi occupation factor. The frequencies $\varepsilon_{m}$ and $\omega_{n}$ are an odd and an even multiple of $\pi / \beta$ respectively. $G_{p}^{0}\left(i \varepsilon_{m}\right)$ is the free electron propagator and $D_{q}^{0}\left(i \omega_{n}\right)$ is the free phonon propagator. By analytical continuation $i \varepsilon_{m} \rightarrow \varepsilon+i \delta$ one gets the retarded self-energy whose real part determines the renormalized electron mass $m_{\text {eff }}$ :

$$
\frac{m_{e f f}}{m_{0}}=\frac{1-\partial \operatorname{Re} \Sigma_{p}(\varepsilon) /\left.\partial \varepsilon\right|_{p=0 ; \varepsilon=-J}}{1+\partial \operatorname{Re} \Sigma_{p}(\varepsilon) /\left.\partial \epsilon_{p}\right|_{p=0 ; \varepsilon=-J}}
$$


where, $\operatorname{Re} \Sigma_{p}(\varepsilon)=\operatorname{Re} \Sigma_{p}^{(1)}(\varepsilon)+\operatorname{Re} \Sigma_{p}^{(2 a)}(\varepsilon)+$ $\operatorname{Re} \Sigma_{p}^{(2 b)}(\varepsilon)+\operatorname{Re} \Sigma_{p}^{(2 c)}(\varepsilon)$, has been obtained from eqs. (4) working out the straightforward but cumbersome double frequency summations and taking the zero temperature limit. The model contains three free parameters: the hopping integral $J$, the zone boundary frequency $\omega_{\pi}=2 \sqrt{K / M}$ which coincides with the zone center optical frequency in the reduced zone scheme, the coupling constant $\alpha^{2} / 4 K$. In Fig. 1, the mass ratio of eq.(5) is plotted versus the adiabaticity parameter $\omega_{\pi} / J$ assuming:(i) a narrow bare band $J$ value, (ii) a weak $e$-ph coupling regime. $m_{e f f}^{(1)}$ denotes the mass renormalization due to the very one-phonon self-energy corrections while $m_{e f f}^{(2)}$ is enriched by the two-phonons scattering processes. Particular care has to be taken in handling the principal values which enter the real self-energy terms. I have used the representation

$$
\text { P.P. }\left(\frac{1}{x}\right)=\lim _{\eta \rightarrow 0} \frac{x}{x^{2}+\eta^{2}}
$$

and achieved numerical convergence by setting $\eta=$ $10^{-4}$ and summing over $200 q$ points in each Brillouin zone. In the extreme adiabatic regime, $\operatorname{Re} \Sigma_{p}^{(1)}(\varepsilon)$ evaluated at the band bottom is much larger than the twophonons terms and the same trend holds for the partial derivatives which enter the mass ratio. Then, $m_{\text {eff }}^{(2)}$ does not differ essentially from $m_{e f f}^{(1)}$ and the renormalization is very poor since scattering by low energy phonons does not enhance the electron mass over the bare band value. As an example, at $\omega_{\pi}=J / 2$, we get (in units meV) $\operatorname{Re} \Sigma_{0}^{(1)}(-J)=1.45, \operatorname{Re} \Sigma_{0}^{(2 a)}(-J)=0.12$, $\operatorname{Re} \Sigma_{0}^{(2 b)}(-J)=-0.17, \operatorname{Re} \Sigma_{0}^{(2 c)}(-J)=-0.5 \cdot 10^{-3}$. At $\omega_{\pi} \simeq 3 J / 5$, the $\operatorname{Re} \Sigma_{0}^{(2 a)}(-J)$ and $\operatorname{Re} \Sigma_{0}^{(2 b)}(-J)$ terms are comparable to $\operatorname{Re} \Sigma_{0}^{(1)}(-J)$ while the $2 c$ diagram is still negligible. Accordingly $m_{\text {eff }}^{(2)}$ starts to get larger than $m_{\text {eff }}^{(1)}$ with an increase of $\sim 15 \%$ in the intermediate $\omega_{\pi} \simeq J$ regime. Although, in this regime, the application of a low order perturbative theory may be questionable, the obtained mass enhancement is likely a signature that quasiparticles with polaronic character may form in the system once multiphonons scattering processes become appreciable. Incidentally we note that a variational study [30] of the SSH model finds in the intermediate regime favourable conditions for the existence of localized polaronic solutions. Fig.2 displays the onephonon effective mass versus $\omega_{\pi} / J$ for two values of $e$ - $p h$ coupling. Setting $J=50 \mathrm{meV}$ we take here an electron band narrower than in Fig.1. Again, the mass increase is relevant only in the intermediate $\omega_{\pi} / J$ - range while the electrons are essentially free in the extreme adiabatic and antiadiabatic regimes. The spike at $\omega_{\pi} \sim \sqrt{2} J$ which dominates the mass behavior is mainly due to scattering by $|q|=\pi / 2$-phonons. This feature is related to the 1D electron and phonon dispersion relations and it may be 
partly suppressed in higher dimensionality. In the $\omega_{\pi} / J$ window which is sensitive to renormalization effects the $e-p h$ coupling parameter slightly affects the values of the one-phonon effective mass. For comparison also the one phonon effective mass of the Holstein-like model is reported on in Fig.2. To point out the role of the e-ph coupling we have replaced the $g^{2}(p, p-q)$ function of the SSH model by a constant $g_{H}^{2}$ without any change to the dispersion relations. Then the Holstein-like model here assumed differs from the "true" Holstein model [15] of diatomic molecules in which purely optical phonons couple to the electrons. We also note that simplified models with dispersionless Einstein phonon spectra would lead to wrong estimates of the ground state properties [16, 31]. Setting $g_{H}^{2}=\alpha^{2} / 4 K \cdot \omega_{\pi}$ with $\alpha^{2} / 4 K=1 \mathrm{meV}$ we obtain a mass behavior similar to that of the SSH model: again we find an abrupt mass increase at $\omega_{\pi} \sim \sqrt{2} J$ (thus confirming its dependence on the 1D dispersion relations rather than on the choice of the coupling function) but the present mass values are generally lower than those predicted by the Holstein model in strong coupling perturbative theory [19]. This suggests that the well known mass enhancement of the Holstein adiabatic and antiadiabatic polaron is due to the multiphononic effects fully captured (for instance) by the Lang-Firsov [32] strong coupling method whereas the details of the short range coupling seem to have little influence on zone center properties such as the effective mass. Conversely one might evaluate the SSH polaron mass in a strong coupling approach to check whether and to which extent the momentum dependent vertex function plays there a peculiar role. Finally, Fig.3 emphasizes that the effective coupling $\alpha^{2} / 4 K$ scarcely affect the mass renormalization in adiabatic and intermediate regimes while a slight mass dependence on $\alpha^{2} / 4 K$ is observed in the antiadiabatic regime with very narrow electron band $J=\omega_{\pi} / 2$. In the latter case however, in the upper portion of the $x$ axis, the dimensionless coupling $\alpha^{2} /(4 K J)$ is larger than one and the perturbative method breaks down. In the intermediate case $\omega_{\pi}=J$ also the two-phonons mass is reported on.

In conclusion, I have assumed a weak coupling perturbative regime and applied low order diagrammatic techniques to the one dimensional Su-Schrieffer-Heeger Hamiltonian in order to compute the electron mass renormalization induced by one- and two-phonons scattering. Tuning the parameter $\omega_{\pi} / J$, the mass behavior has been analysed both in adiabatic, intermediate and antiadiabatic conditions. There is a sizable mass enhancement only in the intermediate and moderately antiadiabatic range whereas the electrons don't drag phonons whose energy is either much smaller or much larger than the electron energy. Hence, no mass enhancement is found in the extreme adiabatic and antiadiabatic regimes. Replacing the momentum dependent vertex function by an Holstein-like coupling constant does not modify substantially the effective mass due to the one phonon self-energy diagram. We emphasize that this result holds in the 
present weak coupling perturbative approach while different conclusions may be drawn in strong coupling theories. The two phonons self-energy corrections introduce an appreciable mass enhancement mostly in the intermediate $\omega_{\pi} \sim J$ regime. This feature can be likely interpreted as an onset of polaronic crossover whose entity and precise location in parameter space requires however computations of multiphonons effects.

FIG. 1. Renormalized masses (in units of bare band electron mass) versus the adiabaticity parameter. $m_{\text {eff }}^{(1)}$ is due to the one phonon self-energy correction. $m_{\text {eff }}^{(2)}$ includes also the two phonons self-energy terms. 
FIG. 2. Renormalized masses (in units of bare band electron mass) as due to the one phonon self-energy correction versus the adiabaticity parameter. Two values of electron-phonon coupling have been chosen. The mass behavior obtained in a Holstein-like model with constant coupling is reported on for comparison.

FIG. 3. Mass renormalization (in units of bare band electron mass) due to one phonon scattering versus the electron-phonon coupling (in $\mathrm{meV}$ ). Three values of the adiabaticity parameter have been chosen. In the intermediate case $J=\omega_{\pi}$ also the effect of the two phonons self-energy correction is displayed.

[1] L.D.Landau, Z.Phys. 3, 664 (1933); L.D.Landau, S.I.Pekar, Zh.Eksp.Teor.Fiz. 16, 341 (1946).

[2] Y.Toyozawa, Prog. Theor. Phys. 26, 29 (1961).

[3] D.Emin, T.Holstein, Phys. Rev. Lett. 36, 323 (1976).

[4] J.T.Devreese, Polarons in Encyclopedia of Applied Physics (VCH Publishers, NY) 14, 383 (1996).

[5] A.S.Alexandrov, N.F.Mott, Rep. Prog. Phys. 57, 1197 (1994).

[6] E.V.de Mello, J.Ranninger, Phys. Rev. B 58, 9098 (1998); ibid., 59, 12135 (1999).

[7] H.Fehske, H.Röder, G.Wellein, A.Mistriotis, Phys. Rev. B 51, 16582 (1995).

[8] E.I.Rashba, in Excitons, Rashba and Sturge eds., NorthHolland, Amsterdam, (1982).

[9] H.De Raedt, A.Lagendijk, Phys. Rev. B 27, 6097 (1983); ibid., 30, 1671 (1984).

[10] G.Kopidakis, C.M.Soukoulis, E.N.Economou, Phys. Rev. B 51, 15038 (1995). 
[11] G.Kalosakas, S.Aubry, G.P.Tsironis, Phys. Rev. B 58, 3094 (1998).

[12] A.H.Romero, D.W.Brown, K.Lindenberg, Phys. Rev. B 59, 13728 (1999); ibid.,60, 14080 (1999).

[13] P.E.Kornilovitch, E.R.Pike, Phys. Rev. B 55, R8634 (1997).

[14] E.Jeckelmann, S.R.White, Phys. Rev. B 57, 6376 (1998).

[15] T.Holstein, Ann. Phys. (N.Y.) 8, 325 (1959).

[16] M.Zoli, Phys. Rev. B 57, 10555 (1998).

[17] M.Zoli, J.Phys.:Condens.Matter 13, 10845 (2001).

[18] Acoustical polarons have been studied also in the framework of the Fröhlich Hamiltonian. See, G.A.Farias, W.B.da Costa, F.M.Peeters, Phys. Rev. B 54, 12835 (1996).

[19] M.Zoli, Phys. Rev. B 61, 14523 (2000).

[20] A.J. Heeger, S.Kivelson, J.R.Schrieffer, W.-P.Su, Rev. Mod. Phys. 60, 781 (1988).

[21] W.P.Su, J.R.Schrieffer, A.J.Heeger, Phys. Rev. Lett. 42, 1698 (1979).

[22] Yu Lu, Solitons and Polarons in Conducting Polymers World Scientific, Singapore (1988).

[23] S.Barisic, Phys. Rev. B 5, 932 (1972).

[24] A.A.Ovchinnikov, I.I.Ukrainskii, G.V.Kventsel, Sov.Phys.Uspekhi 15, 575 (1973).

[25] H.J.Schulz, Phys. Rev. B 18, 5756 (1978).

[26] M.Cococcioni, M.Acquarone, cond-mat/0010164

[27] E.Fradkin, J.E.Hirsch, Phys. Rev. B 27, 1680 (1983)

[28] H.Zheng, Phys. Rev. B 56, 14414 (1997)

[29] G.D.Mahan, Many Particle Physics, Plenum Press, N.Y. (1981) pg.107.

[30] A.La Magna, R.Pucci, Phys. Rev. B 55, 6296 (1997)

[31] M.Capone, W.Stephan, M.Grilli, Phys. Rev. B 56, 4484 (1997)

[32] I.J.Lang, Y.A.Firsov, Sov. Phys. JETP 16, 1301 (1963); Y.A.Firsov, V.V.Kabanov, E.K.Kudinov, A.S.Alexandrov, Phys. Rev. B 59, 12132 (1999). 


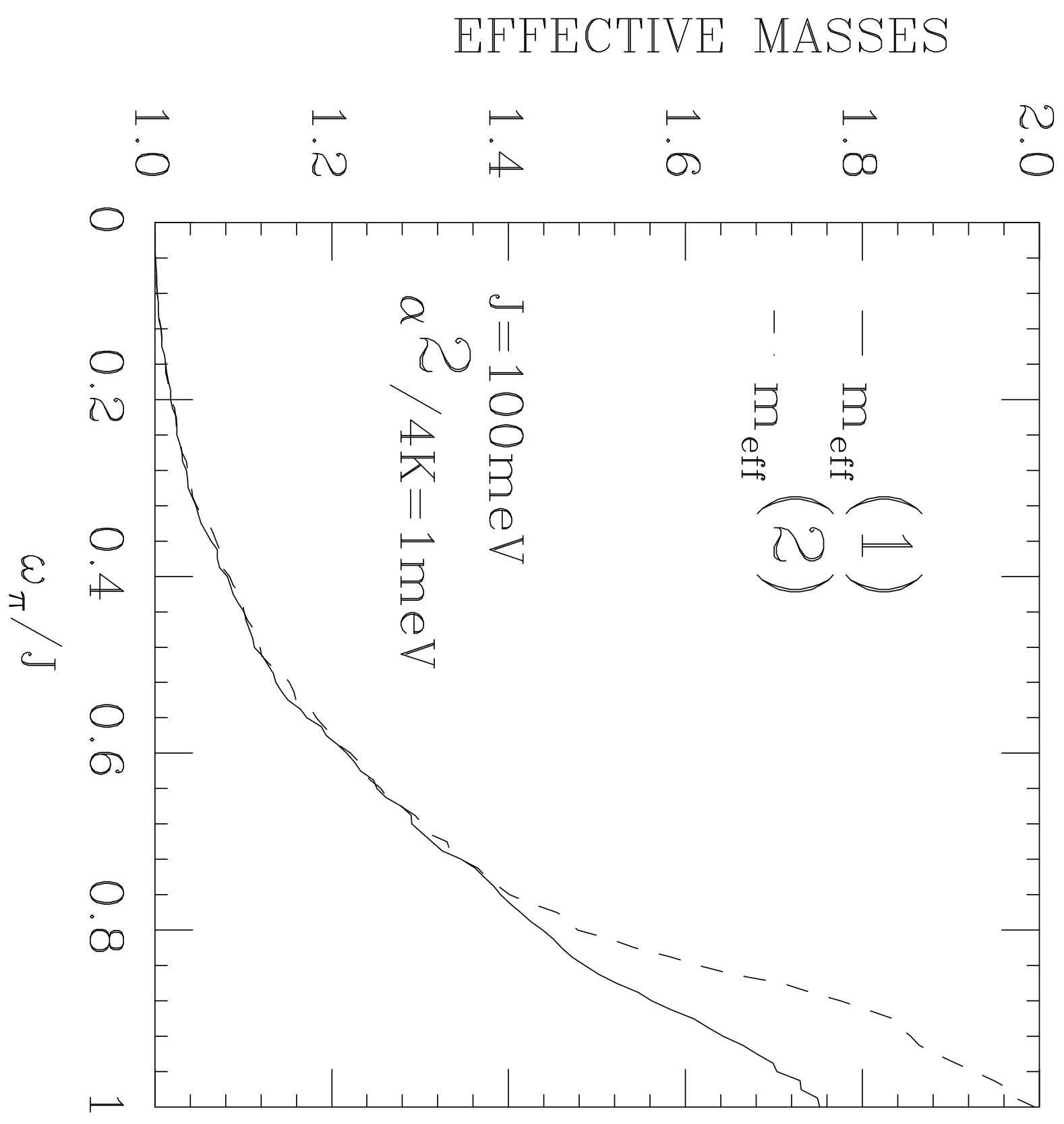




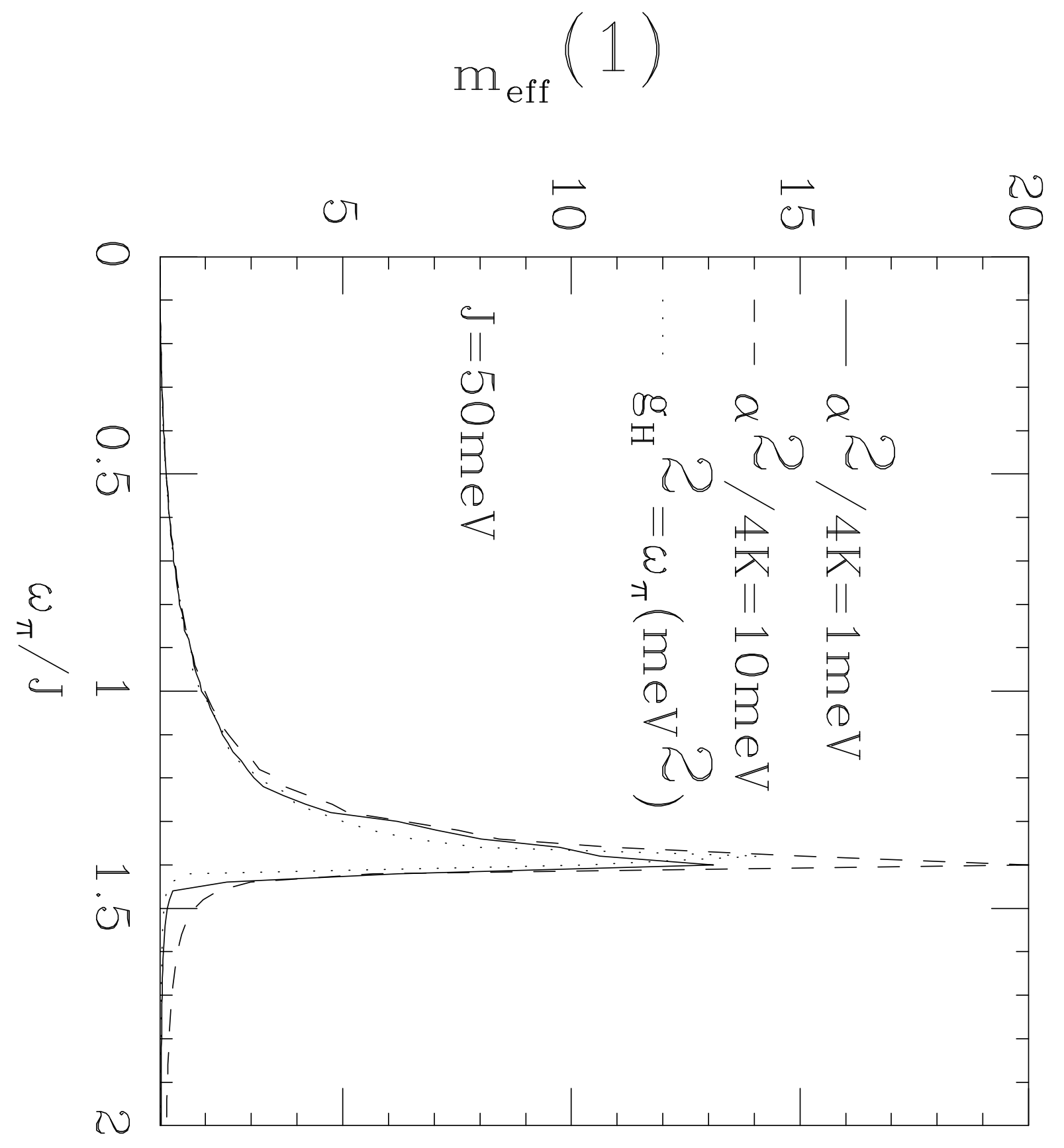




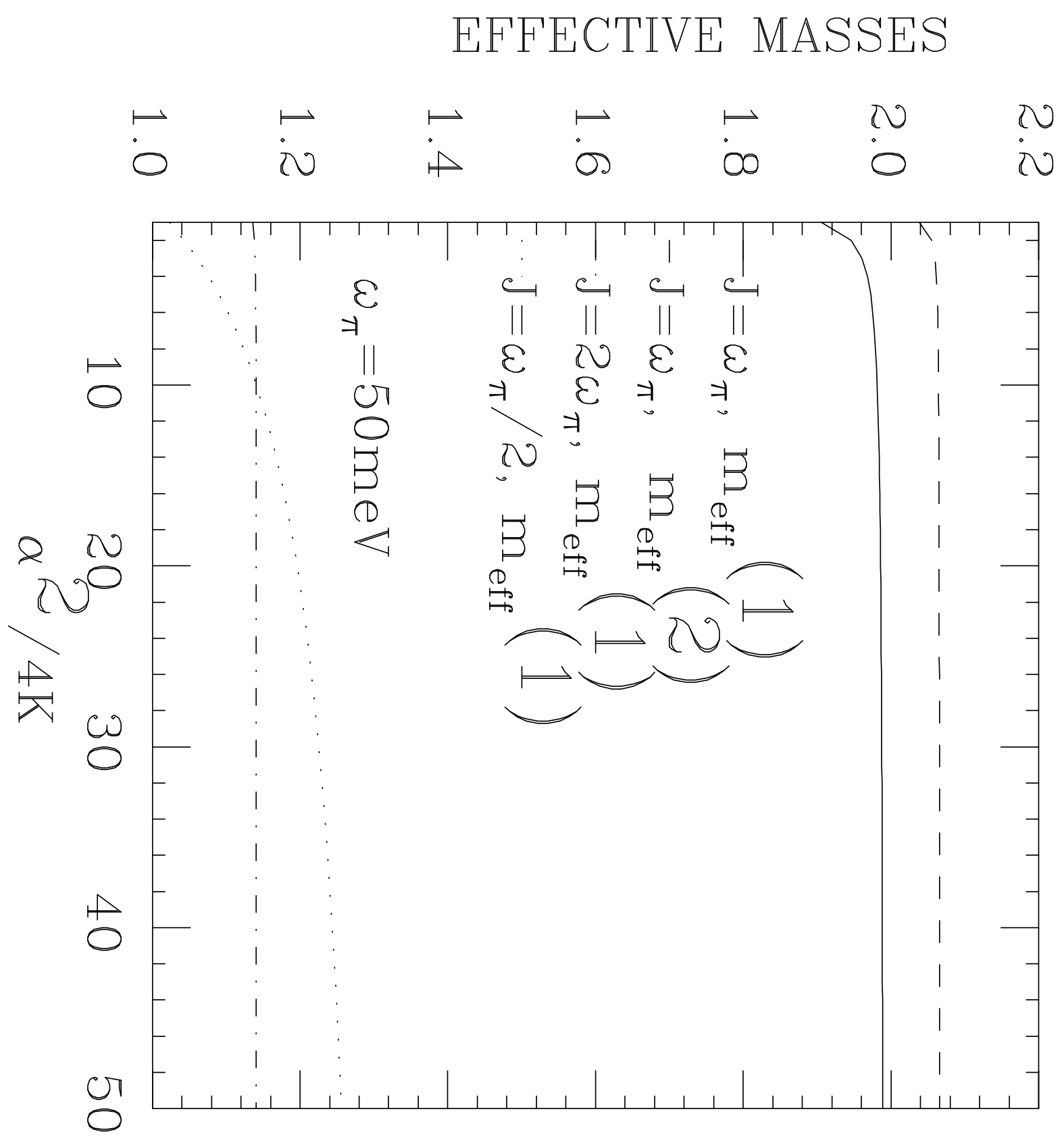

